\title{
Carta ao editor: Alterações Radiográficas Femoropatelares na insuficiência do Ligamento Cruzado Anterior. Rev Bras Ortop 2015;50(1):43-49
}

\author{
Balgovind S. Raja ${ }^{1}$ Sai Gautham Balasubramanian²] \\ ${ }^{1}$ Fortis Escorts Hospital Delhi, Haryana, Índia \\ 2 King Edward Memorial Hospital, Mumbai, Maharashtra, India \\ Endereço para correspondência Sai Gautham Balasubramanian, MS \\ DNB, MRCS, No:31, Aditya nagar, Anand avenue, Madambakkam, \\ Chennai, Tamil Nadu, Índia (e-mail: saigautham90@gmail.com).
}

Rev Bras Ortop 2020;55(4):504.

Caro editor,

Interessamos-nos na leitura de "Alterações Radiográficas Femoropatelares na insuficiência do Ligamento Cruzado Anterior" por de Vasconcelos D. P. et al. ${ }^{1}$ publicado no prestigiado periódico "Revista Brasileira de Ortopedia" no ano de 2015. doi:10.1016/j.rboe.2015.01.005. O objetivo do estudo foi avaliar as alterações radiográficas femoropatelares em 30 pacientes com falha do ligamento cruzado anterior (LCA) usando três parâmetros.

Os autores selecionaram para o estudo 30 pacientes voluntários com lesão unilateral isolada do LCA há $>1$ ano e os compararam com os joelhos contralaterais normais. ${ }^{1} \mathrm{~A}$ altura da patela foi estudada usando o índice de CatonDeschamps, enquanto a inclinação e a translação da patela foram avaliadas, respectivamente, pelo ângulo de inclinação lateral e pelo ângulo de congruência. Embora os critérios de inclusão indiquem que foram incluídos joelhos com uma única lesão do LCA, ao longo de todo o texto nenhuma menção foi feita à integridade do ligamento patelofemoral medial. Na verdade, a deficiência do ligamento patelofemoral medial predispõe ao alinhamento patelofemoral anormal., ${ }^{2,3}$ Os critérios de exclusão também deveriam ter incluído a luxação patelar como um dos critérios, pois ela pode influenciar o objetivo do estudo.

Além disso, o estudo emprega o teste t pareado como ferramenta estatística para descobrir a significância das variáveis. Embora o tamanho da amostra usada seja 30, que é o mínimo necessário para o teste t pareado, os autores do texto mencionam que a inversão do ângulo de inclinação lateral pode ser observada em apenas 2 joelhos $\left(-2,2^{\circ}\right.$ e $\left.-4,8^{\circ}\right)$, o que os torna substancialmente outliers na amostra. Outliers em uma amostra tornam improvável a avaliação do teste $t$ pareado, que é feito com base na distribuição normal. 0 teste Mann-Whitney teria sido uma escolha melhor para esse fim.

Por fim, nenhuma menção ao coeficiente intra- e interobservador foi feita no estudo. $O$ ideal teria sido usar também esses dois parâmetros, o que aumentaria a força do estudo.

Concluindo, a análise estatística do estudo precisa ser verificada e os critérios de exclusão também deveriam incluir joelhos com deficiência do ligamento patelofemoral medial e história de luxação patelar.

Conflitos de Interesses

Os autores não têm conflitos de interesse a declarar.

\section{Referências}

1 de Vasconcelos DP, de Paula Mozella A, de Sousa Filho PG, Oliveira GC, de Araújo Barros Cobra HA. Femoropatellar radiographic alterations in cases of anterior cruciate ligament failure. Rev Bras Ortop 2015;50(01):43-49

2 Reddy KR, Reddy NS. Trochleoplasty and medial patellofemoral ligament reconstruction for recurrent patellar dislocation. Indian J Orthop 2012;46(02):242-245

3 Camp CL, Krych AJ, Dahm DL, Levy BA, Stuart MJ. Medial patellofemoral ligament repair for recurrent patellar dislocation. Am J Sports Med 2010;38(11):2248-2254 recebido

22 de Junho de 2019

aceito

23 de Julho de 2019
DOI https://doi.org/

10.1055/s-0039-1700826.

ISSN $0102-3616$.
Copyright $\odot 2020$ by Sociedade Brasileira License terms de Ortopedia e Traumatologia. Published by Thieme Revinter Publicações Ltda, Rio de Janeiro, Brazil 